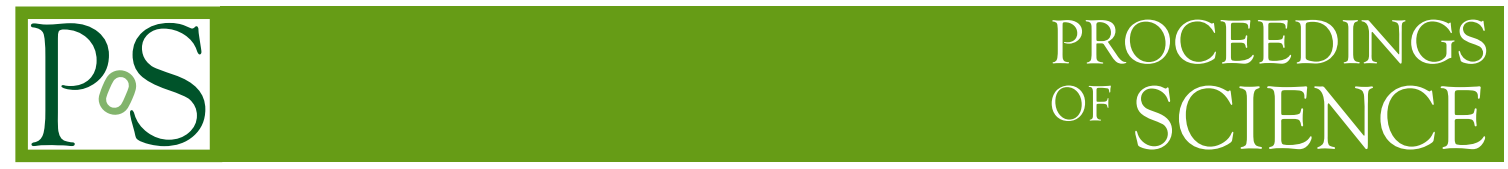

\title{
Ceramic Resistive Plate Chambers for High Rate Environments
}

\author{
Alejandro LASO GARCIA* \\ Helmholtz-Zentrum Dresden-Rossendorf \\ E-mail: a.garcla@hzdr.de \\ Marcus KASPAR \\ Helmholtz-Zentrum Dresden-Rossendorf \\ Burkhard KÄMPFER \\ Helmholtz-Zentrum Dresden-Rossendorf \\ Roland KOTTE \\ Helmholtz-Zentrum Dresden-Rossendorf \\ Lothar NAUMANN \\ Helmholtz-Zentrum Dresden-Rossendorf \\ Richard PESCHKE \\ Helmholtz-Zentrum Dresden-Rossendorf \\ Daniel STACH \\ Helmholtz-Zentrum Dresden-Rossendorf \\ Christian WENDISCH \\ Helmholtz-Zentrum Dresden-Rossendorf \\ Jörn WÜSTENFELD \\ Helmholtz-Zentrum Dresden-Rossendorf
}


Multi-gap resistive plate chambers (RPCs) are proposed to build the Time-of-Flight wall of the Compressed Baryonic Matter experiment with a time resolution better than $80 \mathrm{ps}$. The high fluxes expected at the innermost part of the detector, $\sim 20 \times 10^{3} \mathrm{~cm}^{-2} \mathrm{~s}^{-1}$ require the development of new materials capable of withstanding such fluxes. At Helmholtz-Zentrum Dresden-Rossendorf, several RPC prototypes of $10 \times 10 \mathrm{~cm}^{2}$ and $20 \times 20 \mathrm{~cm}^{2}$ have been built with ceramic plates with bulk resistivities in the range of $10^{9}-10^{10} \Omega \mathrm{cm}$ [1]. They have been tested at the superconducting electron accelerator facility ELBE with $30 \mathrm{MeV}$ electrons. We present the characteristics of the ceramic electrodes and the latest results concerning the performance of these prototypes in electron and protons beams up to fluxes of $10^{6} \mathrm{~cm}^{-2} \mathrm{~s}^{-1}$.

XI Workshop on Resistive Plate Chambers and Related Detectors

5-10 February, 2012

Laboratori Nazionali di Frascati dell'INFN - Frascati (Rome) - Italy

${ }^{*}$ Speaker. 


\section{Introduction}

The Time of Flight Wall (ToF) of the Compressed Baryonic Matter Experiment (CBM) located at the future Facility for Antiproton and Ion Research (FAIR) will be composed of Resistive Plate Chambers (RPCs) [2]. The ToF Wall will be located $10 \mathrm{~m}$ from the target down the beam line and will have a surface of $150 \mathrm{~m}^{2}$. For the most inner part of this detector, simulations predict rates up to $20 \times 10^{3} \mathrm{~cm}^{-2} \mathrm{~s}^{-1}$ for $\mathrm{Au}+\mathrm{Au}$ collisions at $25 \mathrm{AGeV}$. These fluxes are much higher than the typical timing floatglass RPCs can withstand, thus some efforts are needed for developing new materials to work at high rates.

At Helmholtz-Zentrum Dresden-Rossendorf, low-bulk resistivity ceramic materials have been developed which allow RPCs to work in fluxes up to $10^{4}-10^{5} \mathrm{~cm}^{-2} \mathrm{~s}^{-1}$.

\section{Ceramic composites}

The ceramic material developed is a composite of $\mathrm{Si}_{3} \mathrm{~N}_{4} / \mathrm{SiC}$. This material can be tailored to have a bulk resistivity in the range of $10^{7}-10^{12} \Omega \mathrm{cm}$ by modifying the $\mathrm{SiC}$ component.

Various plates with different sizes have been developed. In particular, 12 plates of $10 \times 10 \mathrm{~cm}^{2}$ with a bulk resistivity of about $10^{9} \Omega \mathrm{cm}$ and 30 plates of $20 \times 20 \mathrm{~cm}^{2}$ with an average bulk resistivity of $4 \times 10^{9} \Omega \mathrm{cm}$ (Figure 1) have been inspected and considered as test samples for the needed radiation tolerant RPCs. The bulk resistivity for each plate has been measured in four different and equally spaced points over the surface of the sample. This allows to measure the variation of the bulk resistivity over the surface of each plate. The variation was a factor five at the worst cases.

\section{Detector description}

The architecture employed is a symmetric two-gap structure, giving a total of four gaps for

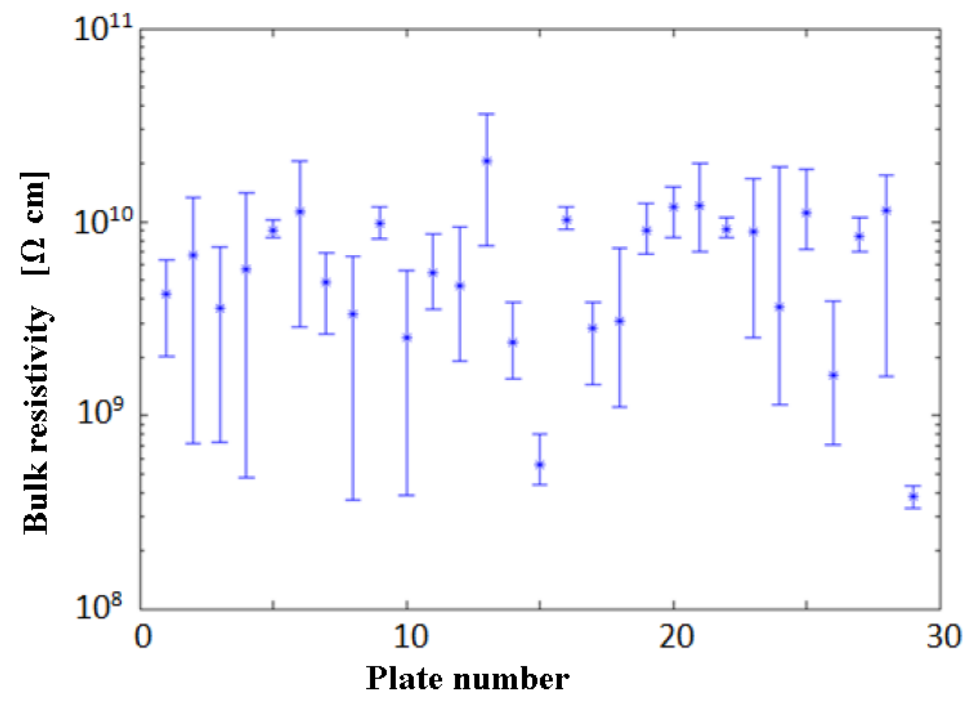

Figure 1: Bulk resistivity for 30 different samples [3]. 


\begin{tabular}{|c|c|c|c|}
\hline prototype & CRPC1 & CRPC2b & CRPC3b \\
\hline size $\left(\mathrm{cm}^{2}\right)$ & $10 \times 10$ & $10 \times 10$ & $20 \times 20$ \\
gap number & 4 & 4 & 4 \\
gap width $(\mu \mathrm{m})$ & 300 & 300 & 250 \\
$\rho(\Omega \mathrm{cm})$ & $\sim 10^{9}$ & $\sim 10^{9}$ & $\sim 4 \times 10^{9}$ \\
separator type & mylar & mylar & fishing line \\
\hline
\end{tabular}

Table 1: Main parameters of ceramic RPC prototypes.

the detector. The high voltage is applied through a dissipative coating made of acrylic paint over a mylar insulator to provide a homogeneous distribution of the high voltage over the whole surface of the plates. The pick-up strips are made of copper and they are placed at the center of the detector mounted on a PCB. The cathode strips are mounted on PCB at the outer part of the detector. The support structure is made of Durostone.

In Table 1 the main parameters of the RPC prototypes tested with electrons and protons are summarized.

\section{In-beam tests}

\subsection{Electron beam test}

The tests with electron beam were performed at the Electron Linac for Beams with high Brilliance and low Emittance (ELBE) at HZDR [4]. The beam delivered by ELBE was of $30 \mathrm{MeV}$ electrons with a pulse duration of $5 \mathrm{ps}$ and a repetition rate of $6.5 \mathrm{MHz}$. However, for detector testing purposes, a special feature of ELBE called 'single electron mode' was used. This mode allows to guarantee than in any pulse delivered by the accelerator there is, at most, one electron.

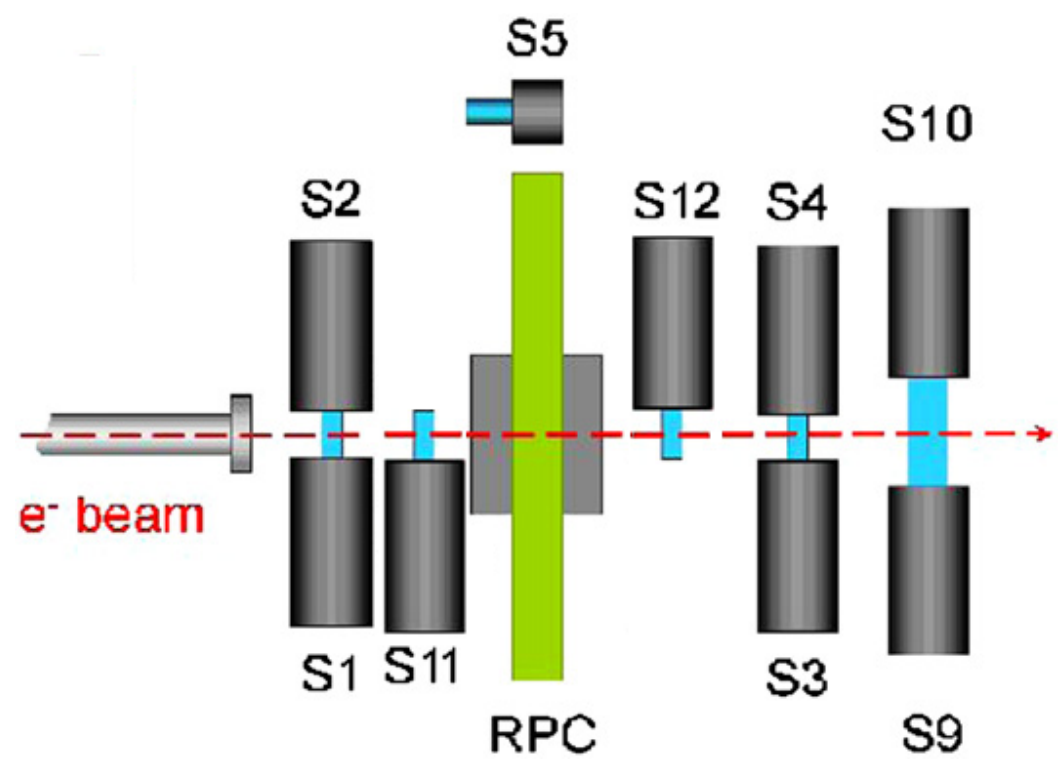

Figure 2: Experimental setup for RPC investigation with $30 \mathrm{MeV}$ electrons at ELBE. 


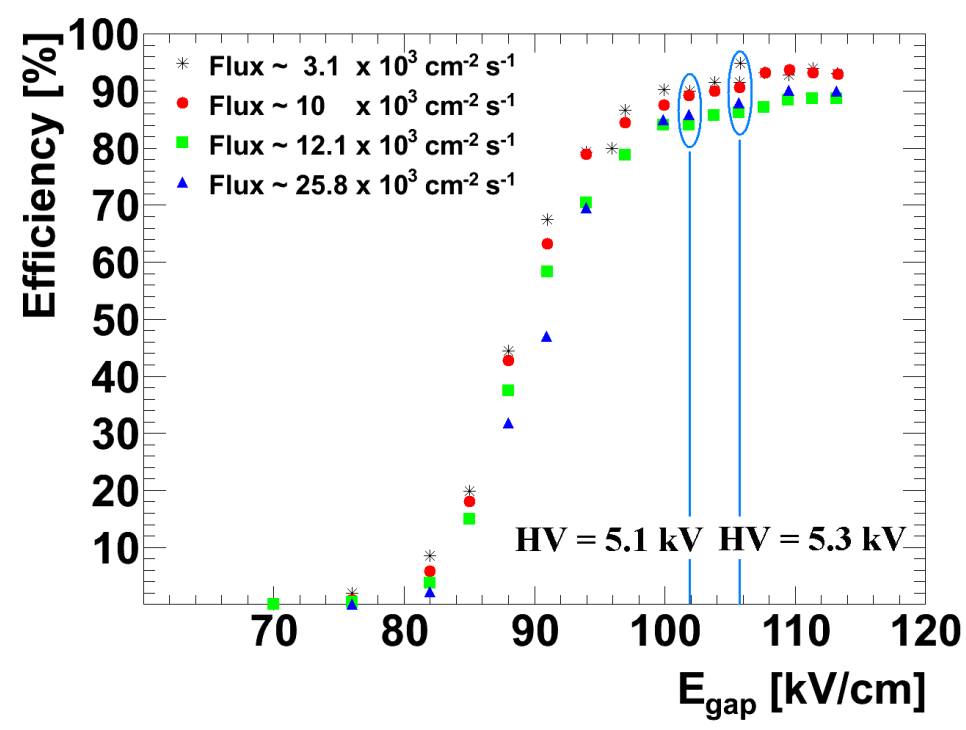

Figure 3: Working curves for a $20 \times 20 \mathrm{~cm}^{2}$ prototype for fluxes up to $25 \times 10^{3} \mathrm{~cm}^{-2} \mathrm{~s}^{-1}$.

With this, it is possible to avoid problems like two particles arriving too close one to another, or more than one particle leaving a signal in the RPC.

The experimental setup is presented in Figure 2. The RPC is mounted on a movable frame which allows for the scanning in the vertical and horizontal position. In the same plane as the RPC, a small scintillator $(\phi=10 \mathrm{~mm}), \mathrm{S} 5$, is mounted. This scintillator is used to measure the beam profile and extract the beam spot. In front of the RPC a scintillator read by two photo tubes, S1 and S2 is placed. Behind the RPC, downstream the beam direction, there is a second scintillator read by two photo tubes, S3 and S4. Two small scintillators $(5 \times 5 \times 2 \mathrm{~mm}), \mathrm{S} 11$ and $\mathrm{S} 12$, are placed immediately before and after, covering a part of one strip of the detector. The reference time is given by the RF signal of ELBE. This provides an excellent start time with $\sigma_{\text {start }} \sim 44 \mathrm{ps}$. The trigger signal is provided by the coincidence $\mathrm{S} 1 \& \mathrm{~S} 2 \& \mathrm{~S} 3 \& \mathrm{~S} 4 \& \mathrm{RF}$. The time information is read by CAEN TDC $1290 \mathrm{~N}$ with a resolution of $24.5 \mathrm{ps} / \mathrm{bin}$. The charge information is also collected by CAEN QDC 965 with a resolution $25 \mathrm{fC} / \mathrm{bin}$. The beamspot has a size of about $14 \mathrm{~cm}^{2}$.

\subsection{Proton beam test}

The proton beam test was perfomed at COSY, Forschungszentrum Jülich, with $2.5 \mathrm{GeV} / \mathrm{c}$ protons. The RPCs were part of a larger setup aimed at testing the different detectors developed by the CBM ToF group. The large prototype was placed with the strips in the horizontal direction while the small prototype was placed inmediately after, with the strips in the vertical direction. The reference time was given by two plastic scintillators placed at the beginning of the setup. These scintillators were readout each by two photo tubes and were set with a relative azimuthal angle of $90^{\circ}$. The trigger was provided by the coincidence of one of the two pairs of these photo tubes with another pair of photo tubes placed at the end of the setup. The beamspot in this case amounts to less than $0.4 \mathrm{~cm}^{2}$. 


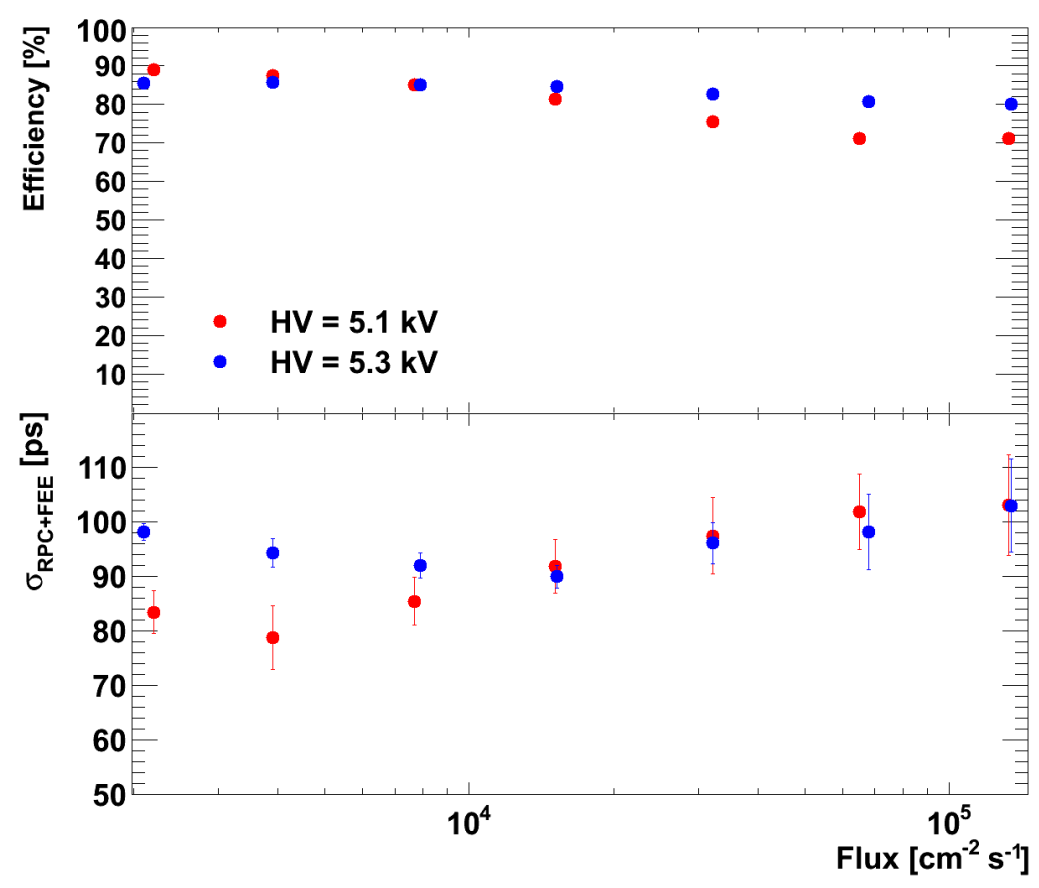

Figure 4: Efficiency and time resolution dependence on the flux for a $20 \times 20 \mathrm{~cm}^{2}$ prototype in a electron beam test.

\section{Discussion of results}

The working point of the detector has been determined measuring the dependence of the efficiency on the electric field in the gap. Two different points were selected corresponding to a voltage applied of $5.1 \mathrm{kV}$ and $5.3 \mathrm{kV}$ respectively. The estimate of the rate capabilities has been done by analysis of the dependence of two main parameters with the particle flux: the efficiency and the time resolution. For the electron beam the result is shown in Figure 3. The efficiency is hereby defined as "hits in the RPC"/"hits in the scintillators" and was extracted from the data files. The time resolution is obtained from gaussian fits of the mean-time spectra of each strip as

$$
\bar{\sigma}=\frac{\sigma_{\text {lower }}+\sigma_{\text {center }}+\sigma_{\text {upper }}}{3},
$$

where $\sigma_{\text {center }}$ is the parameter corresponding to the strip located at the center of the beam, and $\sigma_{\text {lower }}$ and $\sigma_{\text {upper }}$ are the parameters of the nearest neighbors. To present the time resolution of the RPC+FEE, the contribution of the RF is quadratically subtracted.

For the electron beam test the results are presented in Figure 4. It can be seen that the ceramic RPCs present a high efficiency $>70 \%$ up to fluxes of $10^{5} \mathrm{~cm}^{-2} \mathrm{~s}^{-1}$. The time resolution stays below $100 \mathrm{ps}$ in the same range of particle fluxes. It is noteworthy that the read-out strip has not been yet optimized, e.g. is not impedance matched. Thus the time resolution can still be improved to achieve the 70 ps according to the CBM ToF requirements. Also the efficiency can be improved by adding more gas gaps in the detector architecture. 


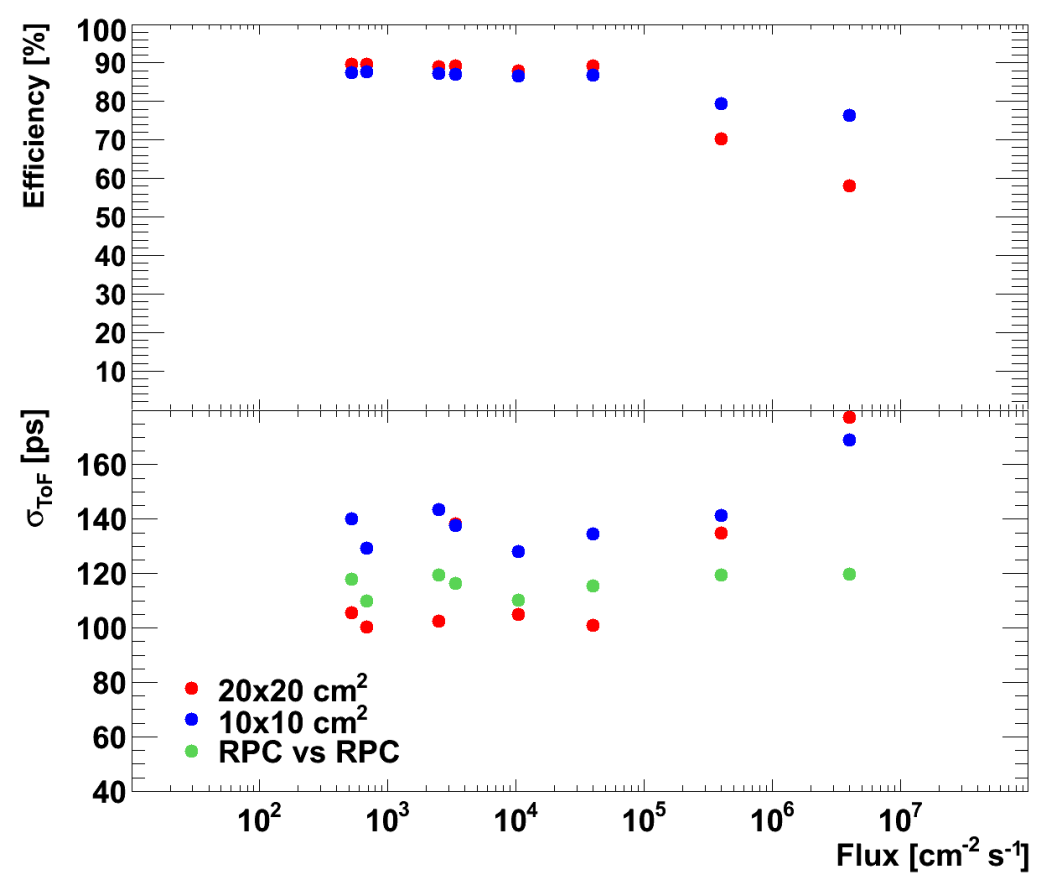

Figure 5: Efficiency and time resolution dependence on the flux for proton beam test with $2.5 \mathrm{GeV} / \mathrm{c}$ protons.

The results obtained for the proton beam can be seen in Figure 5. The results confirm the ones obtained for electron beams. The measurement at the highest flux is orders of magnitude beyond of what can be achieved by glass RPCs. At $\sim 4 \times 10^{6} \mathrm{~cm}^{-2} \mathrm{~s}^{-1}$, the small prototype presents an efficiency of $\sim 78 \%$ and the large one of $\sim 58 \%$. The difference between both detectors can be explained by the difference in the gas gaps widths. The time resolution at this flux amounts to about 120 ps.

\section{Conclusions}

The results obtained for RPC with ceramic electrodes presented in this report show a lot of promise. Thanks to their high radiation tolerance, ceramic materials are well suited for extreme radiation environments. The results obtained for prototypes of different sizes have been shown and discussed. For the highest fluxes $\left(\sim 4 \times 10^{6} \mathrm{~cm}^{-2} \mathrm{~s}^{-1}\right)$ ceramic RPCs present efficiencies of $\sim 78 \%\left(10 \times 10 \mathrm{~cm}^{2}\right.$ prototype $)$ and $\sim 58 \%\left(20 \times 20 \mathrm{~cm}^{2}\right.$ prototype $)$. This fluxes are several orders of magnitude higher than the highest fluxes the typical glass or bakelite RPCs can withstand.

\section{Acknowledgments}

The authors would like to thank the members of the CBM ToF group for the interesting discussions and the collaboration at the COSY beamtime. This work was supported by BMBF 06DR9059. 


\section{References}

[1] L. Naumann et al., Nucl. Instr. Meth. A 628 (2011) 138.

[2] I. Deppner et al., Nucl. Instr. Meth. A 661 (2012) 121.

[3] R. Peschke, Diploma Thesis, 2011, TU-Dresden.

[4] http://www.hzdr.de/db/Cms?pNid=145.

[5] L. Naumann et al., Nucl. Instr. Meth. A 635 (2011) 113. 